\title{
Problemas TEMA I
}

Fernando Favela-Rosales ${ }^{1}$

${ }^{1}$ Instituto Tecnológico Superior Zacatecas Occidente

28 de abril de 2020

\section{Resumen}

En esta tarea se abordan problemas relacionados con los temas: vectores, momento de una fuerza y teorema de Varignon.

\section{Intrucciones}

Resuelva los problemas de acuerdo a la siguiente rúbrica:

- 10\% Entrega un documento en formato PDF mediante el uso de la herramienta Authorea.

- 20\% Plantea ordenadamente los elementos que utilizará para la solución de cada problema.

- 30\% Describe detalladamente el proceso de solución de cada problema. NOTA: incluir respaldo gráfico si así corresponde.

- $\mathbf{4 0 \%}$ Obtiene correctamente la solución de todos los problemas.

1. Para los siguientes pares de vectores calcule analíticamente la suma, el ángulo y la magnitud de la resultante. Además incluya una gráfica con los mismos resultados mediante el uso de Geogebra.

$$
\begin{array}{r}
\vec{A}=11 \hat{i}+7 \hat{j}-3 \hat{k}, \vec{B}=\hat{i}+13 \hat{j}-17 \hat{k} \\
\vec{A}=-23 \hat{i}+7 \hat{j}+15 \hat{k}, \vec{B}=2 \hat{i}-5 \hat{j}-3 \hat{k}
\end{array}
$$

2. Calcule el ángulo y la magnitud de la resultante de la suma de los vectores mostrados en la Figura 1.

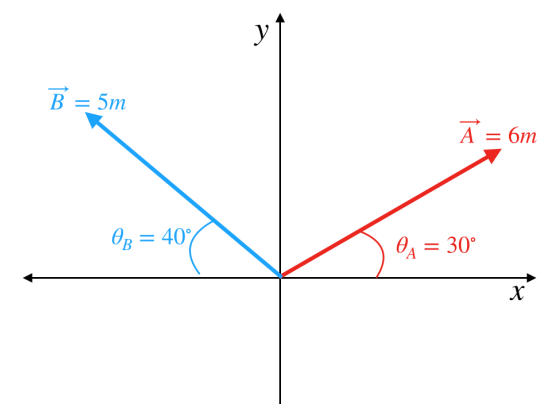

Figura 1: Problema 2.

3. Calcule el momento total con respecto al punto $\mathrm{O}$ debido a las fuerzas del sistema mostrado en la Figura 2.

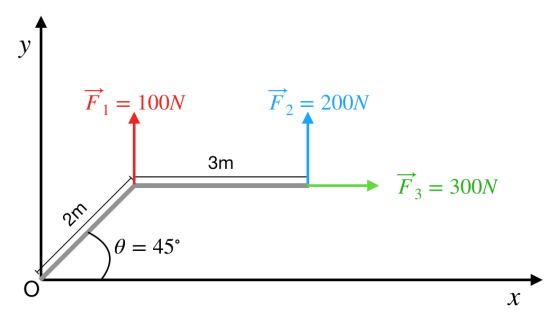

Figura 2: Problema 3.

4. Calcule el momento para el siguiente sistema:

$$
\begin{aligned}
\vec{r} & =(-5 \hat{i}+\hat{j}-7 \hat{k}) \mathrm{m} \\
\vec{F} & =(3 \hat{i}-7 \hat{j}-11 \hat{k}) \mathrm{N}
\end{aligned}
$$

